\title{
NOTIZEN
}

\section{Massenspektrometrische Untersuchung von gasförmigem Chrom(VI)-Oxid}

\section{Harald Schäfer und Klaus Rinke}

Anorganisch-chemisches Institut der Universität Münster (Z. Naturforschg. 20 b, 702-703 [1965]; eingegangen am 5. März 1965)

GLEMSER, MülLer und Stöcker ${ }^{1}$ haben kürzlich den Sättigungsdruck über festem Chrom(VI) -oxid mit der Mitführungsmethode gemessen. Die dort abgeleitete ungewöhnliche Sublimationsentropie $(79 \mathrm{cl} / \mathrm{Mol})$ verdient Interesse. Sie läßt eine komplexe Gaszusammensetzung oder Ungleichgewichte vermuten. Dies veranlaßt uns, eigene massenspektroskopische Beobachtungen mitzuteilen.

Das verwendete Chrom(VI)-oxid war analysenrein und gab das bekannte Debyeogramm ${ }^{2}$. Es wurde im Massenspektrometer (Type CH 4, Atlas MAT Bremen) aus Graphit- und Quarztiegeln verdampft. Die Verdampfungstemperatur lag in der Nähe des Schmelzpunktes $\left(197^{\circ} \mathrm{C}\right)$. Bei einer Beschleunigungsspannung von $2000 \mathrm{~V}$ und einer Elektronenenergie von $50 \mathrm{eV}$ wurden die aus Tab. 1 ersichtlichen Ionen beobachtet.

Die Registrierung erfolgte über einen Sekundärelektronen-Vervielfacher. Unterschiede in den Sekundärelektronen-Ausbeuten sind bei der Intensitätsangabe nicht berücksichtigt. Die Ionen wurden durch Zuordnen von Massenzahlen an Hand eines Eichspektrums und durch Vergleich von berechneten mit gemessenen Isotopenverteilungen identifiziert. Die Auftrittsenergien (unkorrigierte Werte) für die Ionen $\mathrm{Cr}_{3} \mathrm{O}_{7}{ }^{\oplus}, \mathrm{Cr}_{3} \mathrm{O}_{9}{ }^{\oplus}$, $\mathrm{Cr}_{4} \mathrm{O}_{10}{ }^{\oplus}$ und $\mathrm{Cr}_{4} \mathrm{O}_{12}{ }^{\oplus}$ liegen bei $12 \mathrm{eV}$. Die Auftrittsenergie der $\mathrm{Cr}_{2} \mathrm{O}_{4}{ }^{\oplus}$.Ionen liegt dagegen um $19 \mathrm{eV}$. Der weitaus größte Teil der $\mathrm{Cr}_{2} \mathrm{O}_{\mathrm{x}}{ }^{\oplus}$-Ionen entsteht demnach durch Spaltung Cr-reicherer Molekeln. Schon hieraus ist zu schließen, daß primär ringförmige, d. h. Trimere und höhere Molekeln verdampfen, wie sie auch im Dampf von $\mathrm{MoO}_{3}{ }^{3}$ und $\mathrm{WO}_{3}{ }^{4}$ auftreten.
Zumindest ein Teil der beobachteten $\mathrm{Cr}_{3} \mathrm{O}_{7}{ }^{\oplus}$, $\mathrm{Cr}_{4} \mathrm{O}_{10}{ }^{\oplus}$ - und $\mathrm{Cr}_{5} \mathrm{O}_{13}{ }^{\oplus}$-Ionen ist auf $\mathrm{O}$-Abspaltung aus den Gasmolekeln bzw. -Ionen zurückzuführen. Dies wird dadurch deutlich, daß bei geringeren Elektronenenergien die Intensitätsverhältnisse

$$
I\left(\mathrm{Cr}_{\mathrm{x}} \mathrm{O}_{\mathrm{y}}{ }^{\oplus}\right): I\left(\mathrm{Cr}_{\mathrm{x}} \mathrm{O}_{\mathrm{y}-2}{ }^{\oplus}\right)
$$

gegenüber denen bei $50 \mathrm{eV}$ anwachsen (Zerfall als Folge des Elektronenstoßes).

\begin{tabular}{|c|r|c|}
\hline Intensitätsverhältnis & \multicolumn{2}{|c|}{ Elektronenenergie } \\
& $50 \mathrm{eV}$ & $16 \mathrm{eV}$ \\
\hline$I\left(\mathrm{Cr}_{3} \mathrm{O}_{7} \oplus\right): I\left(\mathrm{Cr}_{3} \mathrm{O}_{9} \oplus\right)$ & $2,8: 1$ & $0,3: 1$ \\
$I\left(\mathrm{Cr}_{4} \mathrm{O}_{10} \oplus\right): I\left(\mathrm{Cr}_{4} \mathrm{O}_{12} \oplus\right)$ & $19,8: 1$ & $7,5: 1$ \\
$I\left(\mathrm{Cr}_{5} \mathrm{O}_{13} \oplus\right): I\left(\mathrm{Cr}_{5} \mathrm{O}_{15}{ }^{\oplus}\right)$ & $>7,4: 1$ & $6,5: 1$ \\
\hline
\end{tabular}

Tab. 2. Intensitätsverhältnisse bei verschiedenen Elektronenenergien.

Gleichzeitig treten schon bei einer Elektronenenergie von $16 \mathrm{eV}$ die metastabilen Ionen $\mathrm{Cr}_{4} \mathrm{O}_{9}{ }^{\oplus}, \mathrm{Cr}_{4} \mathrm{O}_{12}{ }^{\oplus}$, $\mathrm{Cr}_{5} \mathrm{O}_{15}{ }^{\oplus}$ auf. Bei $50 \mathrm{eV}$ können noch zusätzlich metastabile $\mathrm{Cr}_{3} \mathrm{O}_{7}{ }^{\oplus}$. und $\mathrm{Cr}_{4} \mathrm{O}_{10}{ }^{\oplus}$-Ionen nachgewiesen werden ${ }^{5}$.

Der zugehörige Zerfallsprozeß lautet:

$$
\mathrm{Cr}_{\mathrm{x}} \mathrm{O}_{\mathrm{y}}{ }^{\oplus}=\mathrm{Cr}_{\mathrm{x}} \mathrm{O}_{\mathrm{y}-2}{ }^{\oplus}+2 \mathrm{O} \text {. }
$$

\begin{tabular}{|c|c|c|}
\hline \multirow[b]{2}{*}{$\begin{array}{c}\mathrm{Cr}_{\mathbf{x}} \mathrm{O}_{\mathrm{y}} \oplus \\
\text { metastabiles } \\
\text { Ion }\end{array}$} & \multicolumn{2}{|c|}{$\mathrm{Cr}_{\mathrm{x}} \mathrm{O}_{\mathrm{y}-2} \oplus$} \\
\hline & $\begin{array}{l}\text { gefundene } \\
\text {,effektive“ } \\
\text { Massenzahl }\end{array}$ & $\begin{array}{l}\text { berechnete } \\
\text {,effektive“" } \\
\text { Massenzahl }\end{array}$ \\
\hline $\begin{array}{l}\mathrm{Cr}_{3} \mathrm{O}_{7} \oplus \\
\mathrm{Cr}_{3} \mathrm{O}_{9} \oplus \\
\mathrm{Cr}_{4} \mathrm{O}_{10} \oplus \\
\mathrm{Cr}_{4} \mathrm{O}_{12} \oplus \\
\mathrm{Cr}_{5} \mathrm{O}_{15} \oplus\end{array}$ & $\begin{array}{c}208 \\
240 \\
307 \\
339 \\
(436)\end{array}$ & $\begin{array}{l}207,8 \\
239,4 \\
306,8 \\
338,6 \\
438,0\end{array}$ \\
\hline
\end{tabular}

Tab. 3. Metastabile Ionen im Massenspektrum des

\begin{tabular}{|c|c|c|c|c|c|c|c|c|c|}
\hline Ion & $\begin{array}{l}\text { rel. } \\
\text { Inten- } \\
\text { sität }\end{array}$ & Ion & $\begin{array}{l}\text { rel. } \\
\text { Inten- } \\
\text { sität }\end{array}$ & Ion & $\begin{array}{l}\text { rel. } \\
\text { Inten- } \\
\text { sität }\end{array}$ & Ion & $\begin{array}{l}\text { rel. } \\
\text { Inten- } \\
\text { sität }\end{array}$ & Ion & $\begin{array}{l}\text { rel. } \\
\text { Inten- } \\
\text { sität }\end{array}$ \\
\hline $\begin{array}{l}\mathrm{Cr} \oplus \\
\mathrm{CrO} \oplus \\
\mathrm{CrO}_{2} \oplus\end{array}$ & $\begin{array}{r}20 \\
14 \\
5\end{array}$ & $\begin{array}{l}\mathrm{Cr}_{2} \mathrm{O}_{2} \oplus \\
\mathrm{Cr}_{2} \mathrm{O}_{3} \oplus \\
\mathrm{Cr}_{2} \mathrm{O}_{4} \oplus \\
\mathrm{Cr}_{2} \mathrm{O}_{5} \oplus\end{array}$ & $\begin{array}{r}3 \\
25 \\
61 \\
5\end{array}$ & $\begin{array}{l}\mathrm{Cr}_{3} \mathrm{O}_{4} \oplus \\
\mathrm{Cr}_{3} \mathrm{O}_{5} \oplus \\
\mathrm{Cr}_{3} \mathrm{O}_{6} \oplus \\
\mathrm{Cr}_{3} \mathrm{O}_{7} \oplus \\
\mathrm{Cr}_{3} \mathrm{O}_{8} \oplus \\
\mathrm{Cr}_{3} \mathrm{O}_{9} \oplus\end{array}$ & $\begin{array}{r}1 \\
11 \\
24 \\
100 \\
2 \\
35\end{array}$ & $\begin{array}{l}\mathrm{Cr}_{4} \mathrm{O}_{7} \oplus \\
\mathrm{Cr}_{4} \mathrm{O}_{8} \oplus \\
\mathrm{Cr}_{4} \mathrm{O}_{9} \oplus \\
\mathrm{Cr}_{4} \mathrm{O}_{10} \oplus \\
\mathrm{Cr}_{4} \mathrm{O}_{11} \oplus \\
\mathrm{Cr}_{4} \mathrm{O}_{12} \oplus\end{array}$ & $\begin{array}{c}0,5 \\
8 \\
5 \\
97 \\
1 \\
5\end{array}$ & $\begin{array}{l}\mathrm{Cr}_{5} \mathrm{O}_{13} \oplus \\
\mathrm{Cr}_{5} \mathrm{O}_{15} \oplus\end{array}$ & $\begin{array}{c}1 \\
0,1\end{array}$ \\
\hline
\end{tabular}
Chrom (VI) -oxids.

Tab. 1. Ionen im Massenspektrum des Chrom(VI) -oxids und relative Intensitäten. Elektronenenergie $50 \mathrm{eV}$.

1 O. Glemser, A. Müller u. U. Stöcker, Z. anorg. allg. Chem. 333, 25 [1964].

2 A. Вуsтröm u. K. A. Wilhelmi, Acta chem. scand. 4, 1131 [1950].

3 J. Berkowitz, M. G. Inghram u. W. A. Chupka, J. chem. Physics 26, 842 [1957].
4 J. Berkowitz, W. A. Chupka u. M. G. Inghram, J. chem. Physics 27, 85 [1957].

5 Zur Methode vgl. C. Brunnee u. H. Voshage: Massenspektrometrie, S. 71, Verlag K. Thiemig, München 1964. 
Die Massenzahlen sind auf das häufigste Isotop bezogen. Im Massenbereich des Pentameren stand kein Eichspektrum zur Verfügung.

Die Instabilität dieser Ionen (mittlere Lebensdauer $\sim 10^{-5} \mathrm{sec}$ ) kann sowohl durch den Elektronenstoß als auch durch thermische Energie verursacht sein.

Berücksichtigt man, daß das Gitter des $\mathrm{CrO}_{3}$ aus Ketten von $\mathrm{CrO}_{4}$-Tetraedern aufgebaut ist ${ }^{2}$ und daß zwischen den Ketten nur schwache Bindekräfte wirksam sind, so kann man sich folgendes Bild machen:

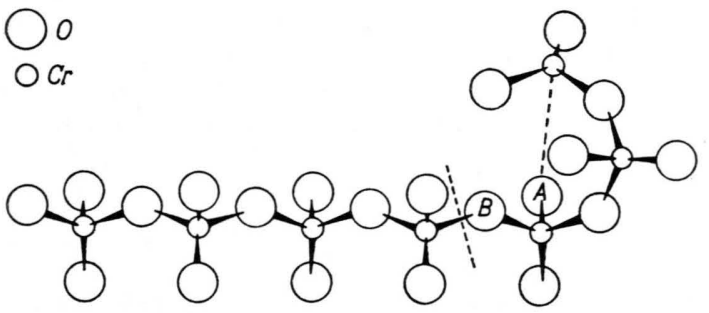

Bei hinreichender thermischer Energie heben sich die Endglieder einer Kette von ihrer Unterlage. Diese beweglichen Kettenenden können im Sinne des Formelbildes einen Ring zum Sauerstoffatom A oder B schließen, wobei die freiwerdende Ringschlußenergie von der gleichen Größenordnung ist wie die zur Spaltung einer $\mathrm{Cr}-\mathrm{O}$-Bindung aufzuwendende Energie. Durch diesen wiederholbaren Vorgang wird der Übergang vom festen in den gasförmigen Zustand sehr erleichtert und die erhebliche Flüchtigkeit des Chrom(VI)-oxids erklärt.

Im Prinzip gilt eine solche Überlegung auch für den Übergang fest $\rightarrow$ flüssig $\rightarrow$ gasförmig.

Diese Vorstellung macht verständlich, daß in der Gasphase vorwiegend Trimere und Tetramere auftreten. Die Abtrennung kleinerer Molekeln aus der kondensierten Phase unterbleibt, weil dabei keine Ringschlußenergie gewonnen würde.

Interessant ist, daß auch Dimere fehlen, obwohl für diese eine koordinative Absättigung durch Kanten-Verknüpfung zweier Tetraeder möglich wäre.

Beim Zusammenstoß der Molekeln mit Elektronen $(12 \mathrm{eV})$ entstehen primär die Ionen $\left(\mathrm{CrO}_{3}\right)_{\mathrm{n}}{ }^{\oplus} \mathrm{mit}$ $n=3,4,5$. Ein erheblicher Anteil hiervon zerfällt z. T. erst nach der Beschleunigung - nach

$$
\left(\mathrm{CrO}_{3}\right)_{n}{ }^{\oplus}=\mathrm{Cr}_{\mathrm{n}} \mathrm{O}_{3 \mathrm{n}-2}{ }^{\oplus}+2 \mathrm{O} \text {. }
$$

Erst bei einer Elektronenenergie $\geqq 19 \mathrm{eV}$ werden ringförmige Molekeln gespalten. Nun treten auch $\mathrm{Cr}_{1} \mathrm{O}_{\mathrm{x}}$ und $\mathrm{Cr}_{2} \mathrm{O}_{\mathrm{y}}$-Teilchen auf. Als Bestandteil einer Gleichgewichtsgasphase wird monomolekulares $\mathrm{CrO}_{3}$ bei $\sim 1600{ }^{\circ} \mathrm{C}$ beobachtet, wenn $\mathrm{O}_{2}$ auf festes $\mathrm{Cr}_{2} \mathrm{O}_{3}$ einwirkt ${ }^{6}$.

Herrn H. Rabeneck sei für die Unterstützung bei der Durchführung dieser Arbeit herzlich gedankt.

6 R. T. Grimley, R. P. Burns u. M. G. Inghram, J. chem. Physics 34, 664 [1961].

\section{Höchstdrudkreaktionen I \\ Entwicklung von Reaktionsgefäßen aus Kunststoff für Drucke $>20000$ atm und Temperaturen von $150-250{ }^{\circ} \mathrm{C}$}

F. Korte, W. Glet und H. Lechner

Organisch-Chemisches Institut der Universität Bonn

(Z. Naturforschg. 20 b, 703-705 [1965]; eingegangen am 7. Mai 1965)

Im Rahmen unserer Arbeiten über chemische Reaktionen unter höchsten Drucken war es für uns von Interesse, Reaktionsgefäße zu entwickeln, die in einer Preßform von $16 \mathrm{~mm}$ Durchmesser wenigstens 20000 atm bis ca. $150{ }^{\circ} \mathrm{C}$ aushielten. Der Druck wurde durch zwei bewegliche Stempel hydraulisch erzeugt. Stempel und Preßform wurden von Ballhausen ${ }^{1}$ für uns konstruiert und gebaut.

1956 beschrieben David und Hamann ${ }^{2}$ eine Druckkapsel aus Polyäthylen mit Teflonverschluß, die bei Zimmertemperatur bis zu 45000 atm in einer zylindrischen Druckform mühelos aushielt. Da unsere Preßformen eine Heizung von außen nicht zuließen, mußten wir Gefäße bauen, die von innen her geheizt werden konnten. Dabei wurden drei verschiedene Arten von Reaktionsgefäßen entwickelt :

1 C. Ballhausen, freischaffender Ing. und freier Mitarbeiter des Mineralog. Inst. d. Universität Bonn, VDI Zeitschr. 105, Nr. 15, 617 [1963].

2 H. G. David, S. D. Hamann, Discuss. Faraday Soc. 22, 119 [1956].
1. Die Wand besteht aus drei Schichten (Abb. 1). Die äußere ist ein $1 \mathrm{~mm}$ dicker Isolierzylinder (A) aus Polyäthylen*, die mittlere ist ein Heizmantel (M), 0,5 mm dick, und besteht aus Teflon mit $20 \%$ Kohlenstoffgehalt ${ }^{* *}$, der oben im Winkel $60^{\circ}$ abgeschrägt ist, um fest an dem entsprechend geformten Deckel (D) anzuliegen. Das eigentliche Reaktionsgefäß bildet den dritten Zylinder (G). Er ist ebenfalls aus RCH 1000

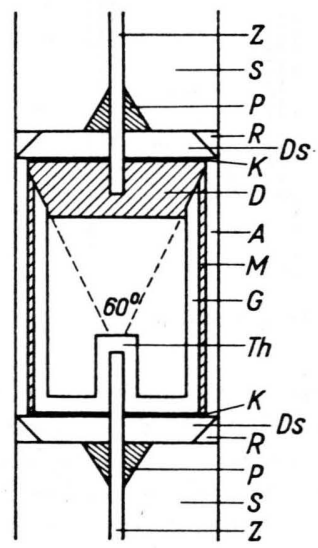

Abb. 1. Reaktionsgefäß.

* Z i e g l e r-Polyäthylen RCH 1000, Firma Ruhrchemie, Oberhausen.

* TFE AL 5, Firma Pampus, Deutsche Gummi- und AsbestGes., Büderich bei Düsseldorf. 